\title{
RIP1 links inflammatory and growth factor signaling pathways by regulating expression of the EGFR
}

\author{
DB Ramnarain ${ }^{1}$, R Paulmurugan ${ }^{2}$, S Park ${ }^{1}$, BE Mickey ${ }^{3,4}$, A Asaithamby ${ }^{5}$, D Saha ${ }^{5}$, MA Kelliher ${ }^{6}$, P Mukhopadhyay ${ }^{7}$, B Banani ${ }^{1}$, \\ CJ Madden ${ }^{3,4}$, PS Wright ${ }^{1}$, S Chakravarty ${ }^{8}$ and AA Habib ${ }^{\star, 1,4,9}$
}

There is considerable interest in understanding how inflammatory responses influence cell proliferation and cancer. In this study, we show that the receptor-interacting protein (RIP1), a critical mediator of inflammation and stress-induced NF- $k B$ activation, regulates the expression of the epidermal growth factor receptor (EGFR). Mouse embryo fibroblasts (MEFs) derived from RIP1 knockout mice express very high levels of the EGFR. Reconstitution of RIP1 ${ }^{-l-}$ MEFs with RIP1 results in a lowering of EGFR levels. RIP1 influences EGFR at the mRNA level by regulating the EGFR promoter. Expression of RIP1 inhibits the EGFR promoter. RIP1 downregulates EGFR expression by interfering with the function of Sp1, which is a key activator of EGFR transcription. RIP1 suppresses Sp1 activity and overexpression of Sp1 reverses RIP1-mediated repression of the EGFR promoter. RIP1 is present both in the cytoplasm and in the nucleus. RIP1 coimmunoprecipitates with Sp1 in vivo and binds directly to Sp1 in vitro. A RIP1 mutant lacking the death domain fails to suppress Sp1 activity and the EGFR promoter, suggesting a critical role for the RIP1 death domain in EGFR regulation. Thus, our study identifies a new link between inflammatory and growth factor signaling pathways mediated by RIP1 and provides insight into the mechanism used by RIP1 to regulate EGFR levels.

Cell Death and Differentiation (2008) 15, 344-353; doi:10.1038/sj.cdd.4402268; published online 16 November 2007

There is a renewed interest in understanding interactions between the immune system and growth factor signaling pathways. ${ }^{1,2}$ Increased expression of growth factor receptors and aberrant growth factor signaling are widespread in cancer. ${ }^{3}$ It is also well known that activation of inflammatory and immune responses may have complex and contextdependent effects on growth factor signaling and cancer. For example, chronic inflammation may play a role in the development and progression of cancer. Conversely, immune responses may also inhibit the progression of cancer and immunotherapy of cancer is aimed at exploiting the immune system to fight cancer. ${ }^{4}$ It has been proposed that generally chronic inflammatory and innate immune responses contribute to the neoplastic process, while adaptive immunity may confer protection against cancer. ${ }^{2}$

The receptor-interacting protein (RIP, RIP1, RIPK1) is widely expressed and is a member of a kinase family that mediates cellular response to stress and includes six other members. $^{5-7}$ RIP1 plays a key role in activation of the transcription factor $\mathrm{NF}-\kappa \mathrm{B}$ and is required for $\mathrm{TNF} \alpha$, TLR3, and DNA damage-induced NF- $\kappa$ B activation. ${ }^{8-12}$ Thus RIP1 is a key component of at least two key signaling pathways mediating the immune response, the TNFR and the TLR3 pathways. RIP1-deficient mice appear normal at birth but fail to thrive and suffer apoptosis in lymphoid and adipose tissues. ${ }^{10}$ RIP1 is expressed constitutively and also inducibly upon T-cell activation. 6,13

Increased expression of the EGFR is common in cancer and there is strong evidence that aberrant EGFR signaling contributes to the pathogenesis of cancer. ${ }^{3}$ The EGFR promoter is well characterized, and a number of factors have been identified that regulate the activity of the promoter in vitro. ${ }^{14,15}$ However, although the receptor has been the focus of intense study for decades, the physiological regulation of EGFR expression in vivo is less well understood. Previous studies have established $\mathrm{Sp} 1$ as a key positive regulator of EGFR transcription and suggest that Sp1 may be required for optimal EGFR transcription. ${ }^{16}$ Indeed, Sp1 is a key regulator of multiple receptor tyrosine kinases, which tend to have GC rich promoters. ${ }^{17}$

\footnotetext{
${ }^{1}$ Department of Neurology, University of Texas Southwestern Medical Center, Dallas, TX, USA; ${ }^{2}$ Molecular Imaging Program at Stanford, Department of Radiology and the Bio-X Program, Stanford University School of Medicine, Stanford, CA, USA; ${ }^{3}$ Department of Neurosurgery, University of Texas Southwestern Medical Center, Dallas, TX, USA; ${ }^{4}$ Annette G. Strauss Center in Neuro-Oncology, University of Texas Southwestern Medical Center, Dallas, TX, USA; ${ }^{5}$ Department of Radiation Oncology, University of Texas Southwestern Medical Center, Dallas, TX, USA; ${ }^{6}$ Departments of Cancer Biology and Medicine, University of Massachusetts Medical School, Worcester, MA, USA; ${ }^{7}$ Department of Internal Medicine, University of Texas Southwestern Medical Center, Dallas, TX, USA; ${ }^{8}$ Department of Psychiatry, University of Texas Southwestern Medical Center, Dallas, TX, USA and ${ }^{9}$ Simmons Comprehensive Cancer Center, University of Texas Southwestern Medical Center, Dallas, TX, USA

*Corresponding author: AA Habib, Department of Neurology, University of Texas Southwestern Medical Center, Mail Code: 8813, 6001 Forest Park ND4.136, Dallas, TX 75390-8813, USA. Tel: 214645 6237; Fax: 214645 6240; E-mail: Amyn.Habib@UTSouthwestern.edu Keywords: receptor interacting protein (RIP1); epidermal growth factor receptor (EGFR); Sp1; growth factor signaling; cross-talk; inflammation

Abbreviations: BSA, bovine serum albumin; EGF, epidermal growth factor; EGFR, epidermal growth factor receptor; EMSA, electrophoretic mobility shift assays; ERK, extracellular signal-regulated kinase; FADD, Fas-associated death domain; IGF-1-R $\beta$, insulin-like growth factor receptor 1 beta; FGFR3, fibroblast growth factor receptor 3; GST, glutathione S-transferase; IKK $\gamma$, Ikappa B kinase gamma; MEF, mouse embryo fibroblast; NEMO, NF- $\kappa$ B essential modulator; NF- $\kappa$ B, nuclear factor kappa B; PDGFR $\beta$, platelet-derived growth factor beta; SOD, superoxide dismutase; RIP1, receptor interacting protein; TLR3, toll-like receptor 3; TNF $\alpha$, tumor necrosis factor alpha; TNFR, tumor necrosis factor receptor; TRADD, TNF receptor-associated death domain

Received 07.3.07; revised 17.9.07; accepted 01.10.07; Edited by J Tschopp; published online 16.11.07
} 
In this study, we identify a role for RIP1, a critical component of inflammatory and immune signaling pathways, as a negative regulator of EGFR expression. A loss of RIP1 from cells results in increased EGFR expression and reconstitution of RIP1 in RIP1 ${ }^{-1-}$ MEFs results in a decrease in EGFR levels. We propose that the mechanism used by RIP1 to inhibit EGFR expression is a direct inhibition of Sp1. We show that RIP1 forms a physical complex with Sp1 and inhibits its activity and investigate the RIP1 domain involved in regulation of EGFR expression.

\section{Results}

RIP1 regulates epidermal growth factor receptor (EGFR) levels. During studies of cytokine signal transduction involving RIP1, we discovered that primary mouse embryo fibroblasts (MEFs) from RIP1 knockout mice expressed a very high level of EGFR compared to wild-type MEFs (Figure 1a). The high levels of EGFR detected in RIP1 ${ }^{-1-}$ MEFs suggests that RIP1 is a negative regulator of EGFR levels. To determine whether the increased EGFR levels influenced EGFR signaling, we tested ERK activation in MEFs. While EGF-induced ERK activation is hard to detect in wild type MEFs, it is robust in RIP1 ${ }^{-1-}$ MEFs, showing that the increased EGFR levels in RIP1 ${ }^{-/-}$MEFs lead to signal amplification (Figure 1b).

Next, we generated immortalized cell lines from $\mathrm{RIP}^{-1-}$ as well as from control RIP1 ${ }^{+/+}$primary MEFs and studied the effect of re-introducing RIP1 into the MEF cell lines lacking RIP1. We used an adenoviral vector-expressing wild type

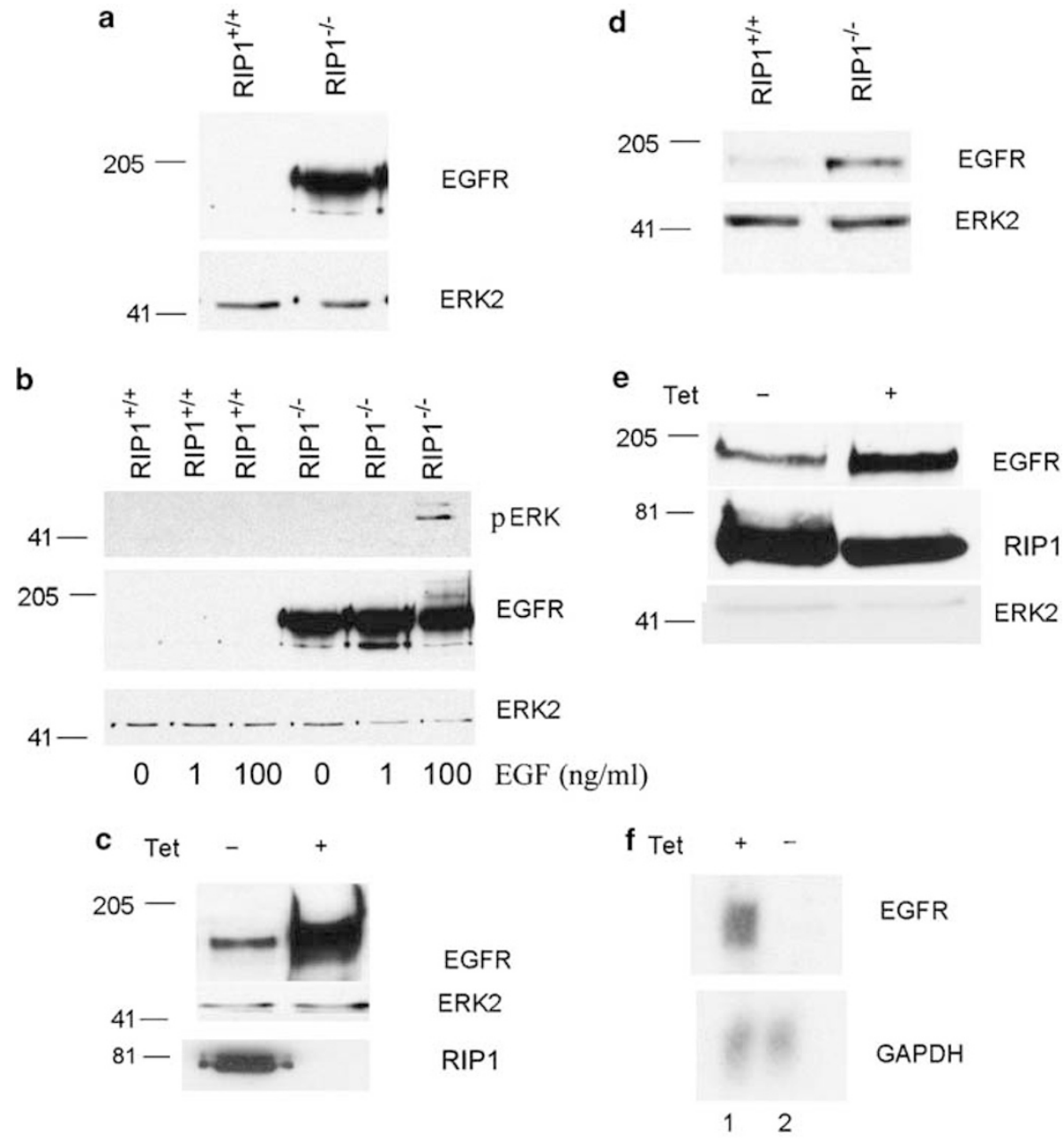

Figure 1 RIP1 regulates EGFR levels. (a) the relative levels of EGFR in mouse embryo fibroblasts (MEFs) in rip $1^{+/+} /$thfr1 ${ }^{-/-}$and rip $1^{-/-}$tnfr $11^{/-}$mouse embryo fibroblasts. Cell lysates from early passage primary MEFs were subjected to Western blot analysis with EGFR antibodies in the upper panel. The blot was stripped and reprobed with ERK2 to show protein loading control (lower panel). There is a substantial increase in EGFR levels in cells lacking RIP1. (b) Cells were serum starved followed by exposure to EGF, preparation of lysates and Western blotting with phospho-ERK antibodies. (c) Reconstitution of RIP1 into RIP1 ${ }^{-1-}$ MEFs results in lowering of EGFR levels. Adenovirus expressing RIP1 under the control of a Tet-off system was infected into MEFs for $48 \mathrm{~h}$ followed by Western blotting with EGFR and RIP1 antibodies (upper and lower panels). Cells exposed to tetracycline do not express RIP1 and have high levels of EGFR expression. The middle panel shows loading. (d) Increased EGFR levels in RIP1 ${ }^{-1-}$ MEFs from a TNFR1 wild-type background compared to wild type MEFs. (e) Overexpression of RIP1 in wild-type MEFs (TNFR1 wild-type background) results in a decrease in EGFR levels. (f) RNA extracted from the corresponding samples was analyzed by Northern blot to demonstrate that RIP1 ${ }^{-1-}$ MEFs have a high level of EGFR mRNA shown in lane 1. When reconstituted with RIP1 (lane 2) the level of EGFR mRNA is decreased suggesting that RIP1 regulates EGFR expression at the transcription level 
RIP1 under the control of a Tet-off system. We find that reconstitution of RIP1 into RIP1 ${ }^{-/-}$cells results in a decrease in EGFR levels (Figure 1c). We find that the level of PDGFR $\beta$ was also increased in RIP1 ${ }^{-1-}$ MEFs and decreased upon reconstitution with RIP1 (Supplementary Figure $1 A$ and $B$ ). However, no difference in the level of either the IGF-IR $\beta$ or FGFR3 was detected either in primary MEFs or upon reconstitution of cells with RIP1 (Supplementary Figure 1CF). This experiment demonstrates that the effect of RIP1 on receptor tyrosine kinase levels is selective.

In the previous experiment we used RIP1 knockout MEFs derived from rip $1^{+/+} / \operatorname{tnfr} 1^{-/-}$and rip $1^{-/-}$tnfr $1^{-/-}$mice. The rip $1^{+/+} /$tnfr $1^{-/-}$MEFs express a very low level of the EGFR which likely results from TNFR1 deficiency. TNFR1 has previously been shown to positively regulate EGFR levels. We also compared EGFR levels in RIP1 knockout MEFs on a wild type TNFR1 background ( $\mathrm{rip}^{+/+} / \mathrm{tnfr}^{+/+}$and rip $1^{-/-}$tnfr $1 /^{+/+}$) as seen in Figure $1 \mathrm{~d}$ and find that EGFR levels are increased in these RIP1 knockout cells as well. Furthermore, overexpression of RIP1 in wild type MEFs $\left(\right.$ rip $1^{+/+} /$tnfr $\left.1^{+/+}\right)$also results in a decrease in EGFR levels (Figure 1e). Thus, RIP1 regulates the EGFR level whether or not TNFR1 is expressed.

RIP1 could regulate EGFR at the level of mRNA or protein. We conducted Northern blotting using RNA extracted from $\mathrm{RIP}^{-1-}$ MEFs in lane 1, or reconstituted RIP1 ${ }^{-1-}$ MEFs with RIP1 in lane 2 (Figure 1f) and find that RIP1 influences EGFR at the mRNA level. We also compared the level of EGFR mRNA in rip $1^{+/+} /$tnfr $^{+/+}$MEFs and rip $1^{+/+} /$tnfr $^{-/-}$ MEFs. As can be seen in Supplementary Table 1, and consistent with the Western blot result, there is more EGFR mRNA in rip $1^{+/+} /$tnfr $^{+/+}$MEFs.

Next, we investigated if there is a correlation between the levels of RIP1 and EGFR in a panel of breast cancer cell lines. As can be seen in Figure 2, there is an inverse correlation between the RIP1 and the EGFR levels in most of the cell lines examined. This experiment suggests that RIP1 may regulate EGFR levels in cancer.

RIP1 is a strong repressor of the EGFR promoter. Next, we studied the influence of RIP1 on the function of the EGFR promoter. 293 cells were transiently transfected with the wild type EGFR promoter driving luciferase gene (pER1-LUC) along with either empty vector or wild-type RIP1 in a mammalian expression vector (Figure 3a). RIP1 significantly $(P<0.001)$ represses the activity of the EGFR promoter in these cells. The same experiment performed in MEFs showed similar results $(P<0.01$, Figure $3 b)$. Western blots were performed to confirm expression of RIP1 (data not shown). These experiments suggest that RIP1 is a strong repressor of the EGFR promoter. In addition, the activity of the EGFR promoter is increased in $\mathrm{RIP}^{-1-}$ cells compared to $\mathrm{RIP} 1^{+1+}$ MEFs $(P<0.001)$ as shown in Figure 3c. Figure $3 d$ shows that expression of RIP1 inhibits the EGFR promoter in a dose-dependent manner in 293 cells. Expression of RIP1 was confirmed by Western blotting and quantitated by densitometry as shown in Figure 3e. To test whether other components of the NF- $\kappa \mathrm{B}$ signaling network had a similar effect, we expressed IKK $\gamma /$ NEMO along with the EGFR promoter. IKK $\gamma / \mathrm{NEMO}$ does not suppress activity of
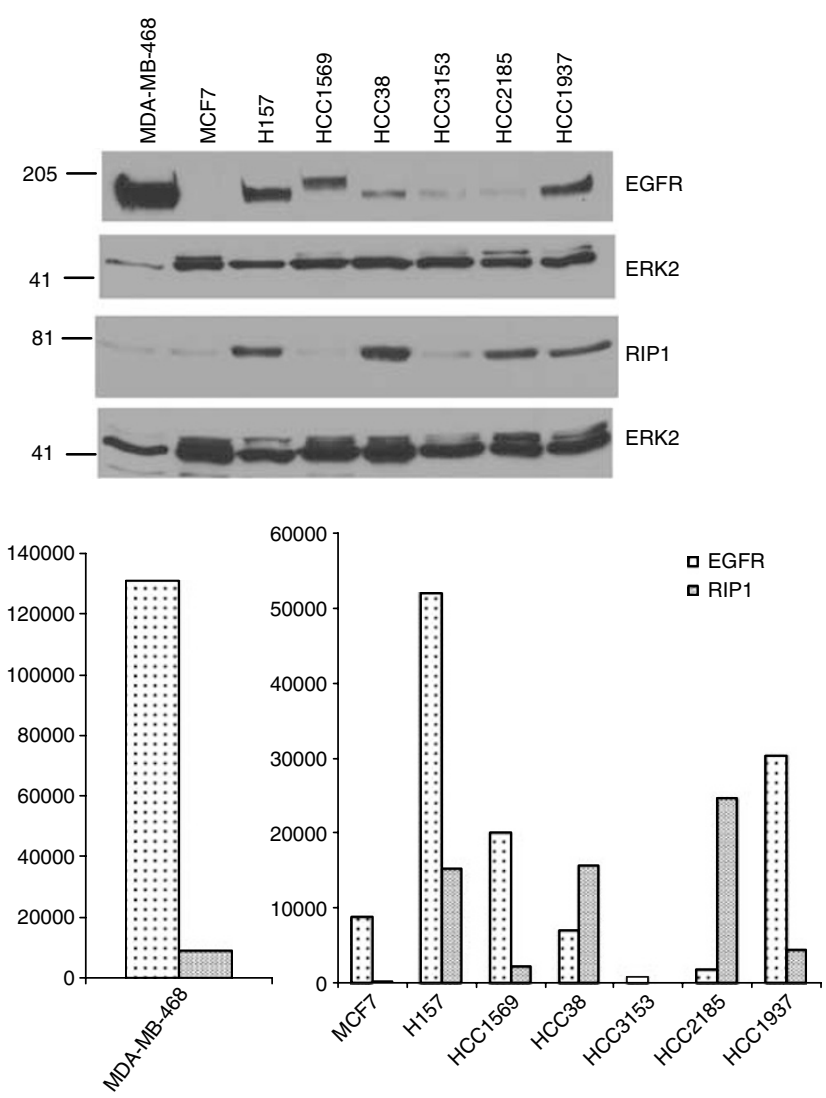

Figure 2 Correlation of RIP1 and EGFR levels in a panel of breast cancer cell lines. Western blot showing EGFR and RIP1 levels in eight breast cancer cell lines. The lower panel shows densitometry values (intensity $/ \mathrm{mm}^{2}$ normalized for ERK2 as a loading signal) as a bar diagram. The data is shown in two bar diagrams because of the huge disparity in EGFR versus RIP1 levels in MDA-MB-468 cells. There is an inverse correlation between EGFR and RIP1 levels in most of the cell lines examined

the EGFR promoter $(P=0.24)$, whereas in the same experiment RIP1 suppressed the EGFR promoter $(P<0.05)$ as shown in Figure $3 f$, suggesting that the effect on the EGFR promoter may be relatively specific to RIP1. Next, we tested the effect of increasing IKK $\gamma / \mathrm{NEMO}$ expression on the EGFR promoter (Supplementary Figure 2). We find that while IKK $\gamma / \mathrm{NEMO}$ overexpression strongly suppresses $\mathrm{TNF} \alpha$-induced $\mathrm{NF}-\kappa \mathrm{B}$ activation as has been reported previously, ${ }^{18}$ it fails to inhibit the EGFR promoter even at a high level of expression.

RIP1 is composed of a kinase domain, an intermediate domain, and a death domain ${ }^{6}$ (Supplementary Figure 3). To identify which domain of RIP1 mediates its repression of the EGFR promoter, we conducted reporter assays with the EGFR promoter ( $p E R 1-L U C)$, cotransfected with either wildtype RIP1 or RIP1 mutants lacking either the kinase, intermediate, or the death domain. As can be seen in Figure $4 a$, deletion of the death domain completely abolished the ability of RIP1 to suppress the EGFR promoter suggesting that the death domain plays a critical role in EGFR regulation. Expression of the wild type and mutant RIP1 is shown in Figure 4b. 

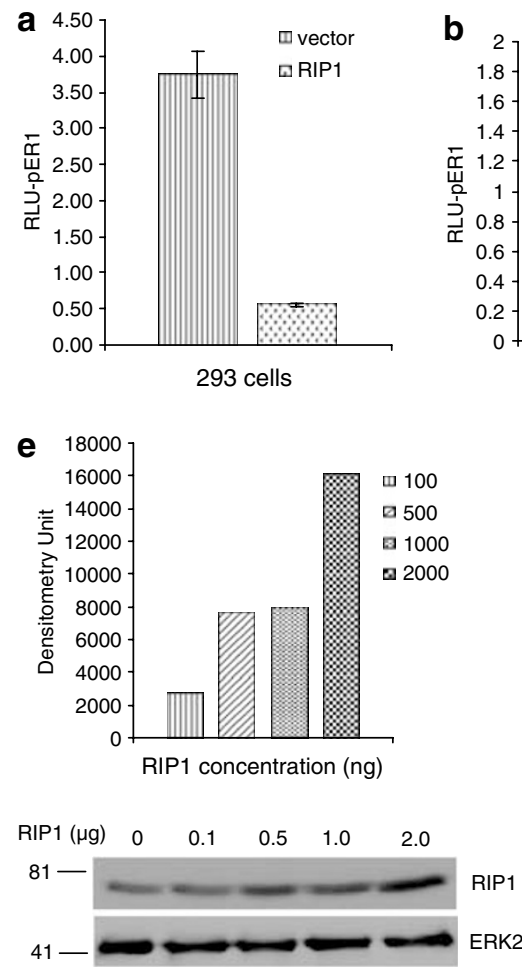
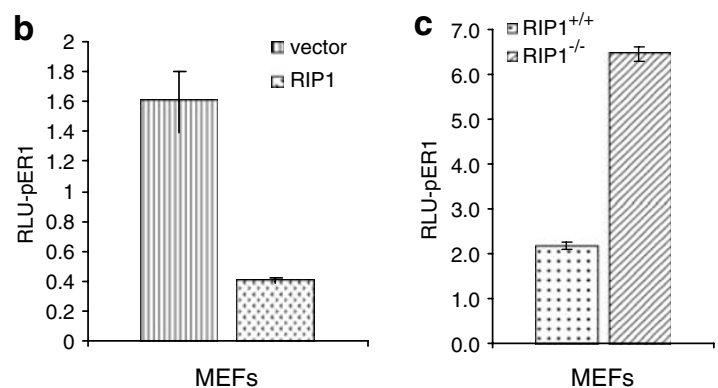

d
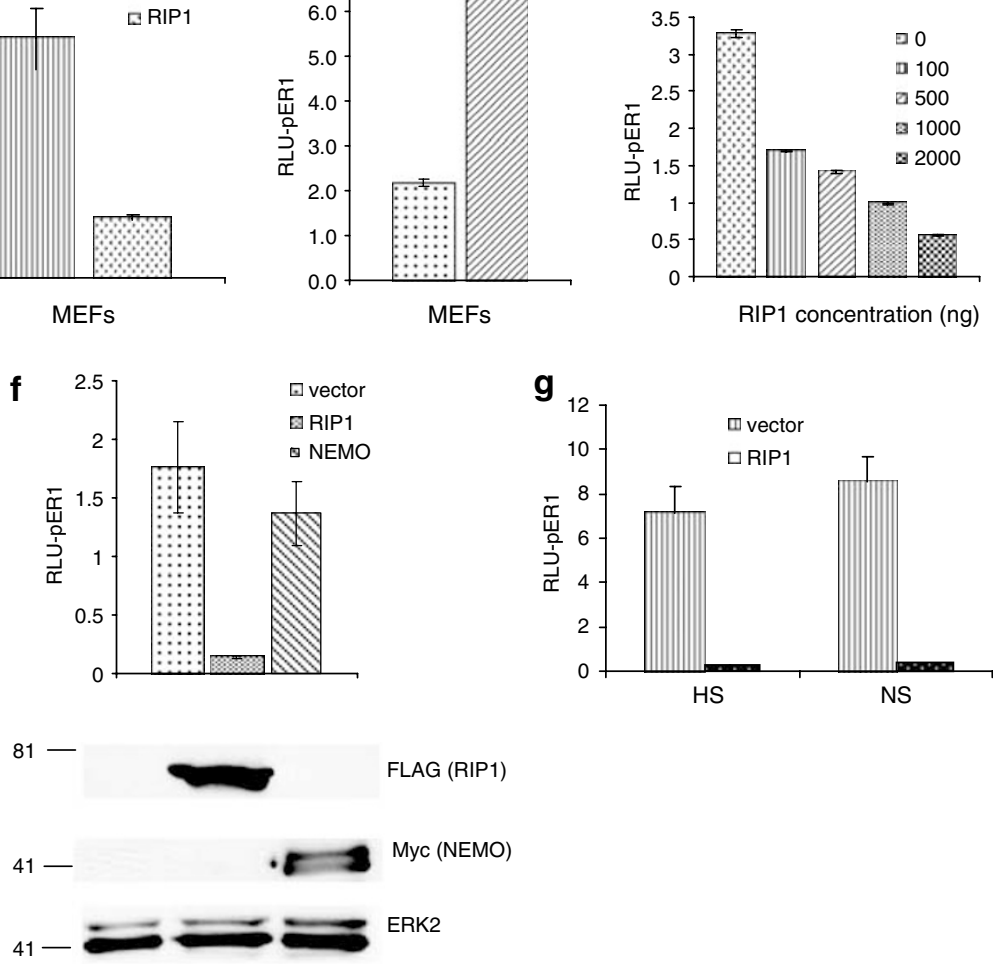

Figure 3 RIP1 is a powerful repressor of EGFR transcription. (a) 293 cells were cotransfected with the full-length EGFR promoter linked to firefly luciferase ( $\mathrm{pER} 1$-LUC) along with either empty vector or wild-type RIP1. (b) Shows the same experiment in RIP1 ${ }^{-l-}$ MEFs. (c) The activity of the EGFR promoter is higher in RIP1 ${ }^{-1-}$ MEFs compared to RIP ${ }^{+1+}$ MEFs. (d) Shows that RIP1 suppresses activity of EGFR promoter in luciferase assays in a dose-dependent manner. (e) Expression of RIP1 by Western blotting. The upper panel shows the increasing RIP1 levels in the Western blot by densitometry (value of endogenous RIP1 was subtracted). (f) NEMO/IKK $\gamma$ fails to suppress the activity of the EGFR promoter while RIP1 suppresses it. The experiment was conducted as in (a). The lower panel shows expression of FLAG-tagged RIP1 and Myctagged NEMO/IKK $\gamma$. (g) 293 cells were transfected with RIP1 along with either a full-length promoter-driving luciferase ( -1109 to -16, HS/RIP1) or a truncated EGFR promoter-driving luciferase (NS/RIP1, -153 to -19$)$. The ability of RIP1 to repress activity of the fragment containing sequences from -153 to -19 is comparable to the wildtype promoter. Experiments were repeated three times in triplicate. Renilla luciferase was used as an internal control in all experiments. Error bars represent S.E.M. of triplicate determinations. RLU, relative luciferase unit

RIP1 inhibits Sp1 activity. Since RIP1 does not have a DNA binding domain, it is likely to exert its effect indirectly. To identify the transcriptional regulators involved, we used a strategy based on determining the effect of RIP1 on EGFR promoter deletion mutants in reporter assays. We used a fulllength EGFR promoter spanning the sequences from -1109 to $-16(\mathrm{HS})$ and a deletion mutant that contained sequences from -153 to -19 (NS) ${ }^{19}$ shown in Supplementary Figure 3. This fragment has previously been shown to retain most of the activity of the promoter. $^{20}$ We found that RIP1 significantly suppressed both the wild type $(P<0.01)$ and the deletion mutant $(P<0.001)$ equally (Figure $3 \mathrm{~g})$. This suggested that the region between -153 and -19 was sufficient to mediate the repressive effects of RIP1. Consistent with a previous study, ${ }^{20}$ we were not able to examine the effect of RIP1 on smaller deletion fragments in this region, because of low basal activity of these mutants.

A number of studies have shown that the Sp1 is a key activator of EGFR transcription. Since deletion analysis of the EGFR promoter did not provide clear information about how RIP1 may repress the EGFR promoter, we investigated whether RIP1 influences activity of the EGFR promoter via an inhibition of Sp1. First, we tested whether Sp1 activates the
EGFR promoter. Consistent with previous studies, we found that Sp1 significantly $(P<0.001)$ activates the EGFR promoter (Figure 5a). In this experiment, we transfected pER1-LUC along with either Sp1 or empty vector into 293 cells. Sp1 expression induces a substantial activation of the EGFR promoter. Next, we investigated whether RIP1 influences Sp1 activity. 293 cells were transfected with a Sp1-LUC construct along with either empty vector or wild-type RIP1. This Sp1LUC construct has three Sp1-binding sites from the SV40 promoter. We find that, in these cells, RIP1 strongly $(P<0.01)$ represses Sp1 activity (Figure 5b) in reporter assays.

Sp1 reverses RIP1-mediated repression of the EGFR promoter. Our hypothesis that the mechanism used by RIP1 to downregulate EGFR expression is via inhibition of Sp1 activity is further supported by experiments showing that expression of Sp1 rescues the RIP1-mediated repression of the EGFR promoter. In the experiment shown in Figure 5c, the pER1-LUC was transfected into 293 cells along with RIP1 and either empty vector or Sp1. Expression of Sp1 blocks the inhibition of the EGFR promoter induced by RIP1. Since Sp1 expression activates the EGFR promoter, the reversal of RIP1-mediated repression of the EGFR by Sp1 by itself does 

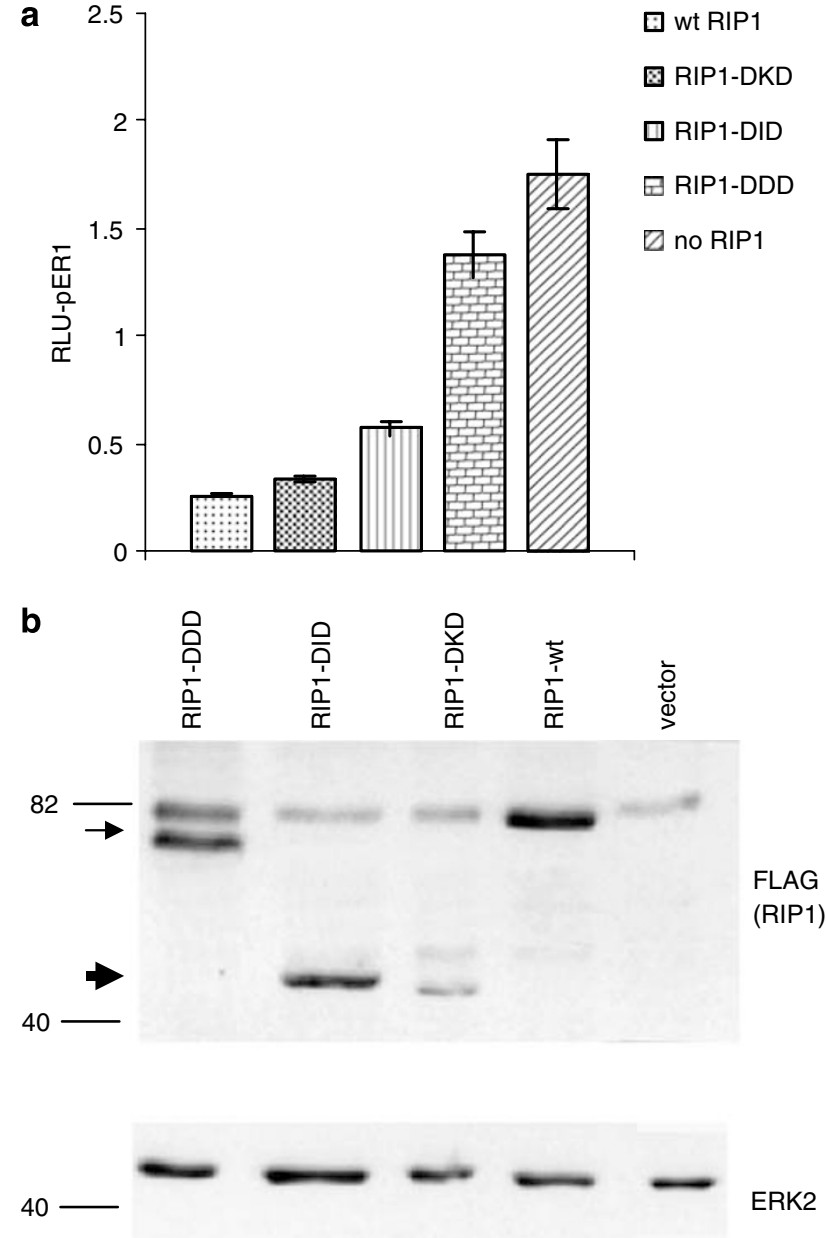

Figure 4 (a) The ability of wild-type RIP1 or various RIP1 mutants to inhibit the wild-type EGFR promoter in reporter assays. The RIP1 mutants used here are deletion of the kinase domain (RIP1-DKD), deletion of the intermediate domain (RIP1-DID), or deletion of the death domain (RIP1-DDD). 293 cells were transfected with wild-type RIP1 or mutant RIP1 along with pER1-LUC. We also transfected pER1-LUC with an empty vector as a control. Luciferase activity was measured after $24 \mathrm{~h}$. Deletion of the death domain results in a complete loss of the ability to suppress the luciferase expression. Renilla luciferase was used as an internal control in all experiments. Error bars represent S.E.M. of triplicate determinations. (b) Shows expression of FLAG-tagged wild-type RIP1 or various mutants in 293 cells by Western blotting with FLAG antibodies. A non-specific band is detected by M2 FLAG antibodies in all lysates that comigrates with the wild type FLAG-RIP1 and can be seen in the empty vector lane also. The RIP1 deletion mutants are smaller in size and migrate faster. The thin arrow points to RIP1 DDD-FLAG while the thicker arrow points to RIP1 DID-FLAG and RIP1 DKD-FLAG. The lower panel shows loading control. Experiments were repeated three times in triplicate

not prove this mechanism. However, taken in conjunction with our other findings, this experiment supports the hypothesis that RIP1 inhibits the EGFR promoter via inhibition of Sp1. For example, we find that Sp1 activity is increased in $\mathrm{RIP1}^{-/-}$MEFs compared to RIP1 ${ }^{+/+}$MEFs $(P<0.001)$ as shown in Figure 5d. Finally, consistent with our previous results showing a requirement for the RIP1 death domain for repression of the EGFR promoter, we find that the RIP1 mutant lacking the death domain also fails to inhibit Sp1 activity (Figure 5e). Expression of wild type and mutant RIP1 is shown in Supplementary Figure 4.

RIP1 expression impairs Sp1 binding to DNA. Having determined that RIP1 inhibits Sp1 activity in reporter assays, we investigated whether RIP1 expression interferes with Sp1 binding to DNA. Electrophoretic mobility shift assays were performed with nuclear extracts from RIP1 ${ }^{-1-}$ MEFs or RIP1 ${ }^{-/}$MEFs expressing RIP1 incubated with labeled oligonucleotide probes bearing a Sp1 binding site. As can be seen in Figure 6, expression of RIP1 significantly impairs DNA binding. The binding to Sp1 probe is specific because a probe with mutations in the Sp1 sequence failed to bind nuclear proteins. Incubation of nuclear extracts with anti-Sp1 antibody but not control antibodies resulted in Sp1 supershift, confirming presence of Sp1 in the complex with Sp1 oligonucleotide.

RIP1 in the nucleus. Next, we considered the possibility that RIP1 may physically interact with Sp1 and inhibit its activity. Sp1 is primarily nuclear while RIP1 has previously been localized only to the membrane and cytosolic compartment. ${ }^{21}$ We studied the localization of RIP1 in wildtype MEFs and HeLa cells by cell fractionation as well as immunofluorescent staining. As is shown in Figure 7a, although much of the cellular RIP1 is cytosolic, RIP1 can also be detected in nuclear fraction. Sp1 is mostly detected in the nuclear extract. Next, we investigated the localization of RIP1 by immunofluorescent staining. As can be seen in Figure 7b-d, RIP1 is present in the nucleus. Thus, endogenous RIP1 can be detected in the nucleus in two different cell types.

The localization of RIP1 and Sp1 was further studied by confocal microscopy as shown in Figure 7c and d. Our results show that RIP1 is unequivocally present in the nucleus. Furthermore, RIP1 and Sp1 are in close proximity and overlap and touch in multiple areas (Figure 7c and d). However, the Pearson coefficient for the actual colocalized voxels is low (0.0168). Thus, we are unable to demonstrate statistically that RIP1 and Sp1 colocalize using this technique. Thus, although RIP1 and Sp1 bind directly in vitro (see below), it remains possible that the interaction in vivo is facilitated by some other protein(s).

RIP1 forms a complex with Sp1 in cells. To test whether RIP1 interacts physically with Sp1, we transfected an HAtagged Sp1 construct into 293 cells. Immunoprecipitation of whole cell lysates with RIP1 antibodies was followed by immunoblotting with $\mathrm{HA}$ or Sp1 antibodies. We can detect RIP1 and Sp1 coimmunoprecipitation in these cells if Sp1 is overexpressed, as shown in Figure $8 a$ and b. In further experiments, we cotransfected a Myc-tagged RIP1 construct along with Sp1-HA into 293 cells. Immunoprecipitation of whole-cell lysates with Myc antibodies was followed by immunoblotting with HA antibodies as shown in Figure 8c. Thus, immunoprecipitation of endogenous RIP1 with RIP1 antibodies or transfected Myc-tagged RIP1 with Myc antibodies results in coimmunoprecipitation of coexpressed Sp1-HA. 
a
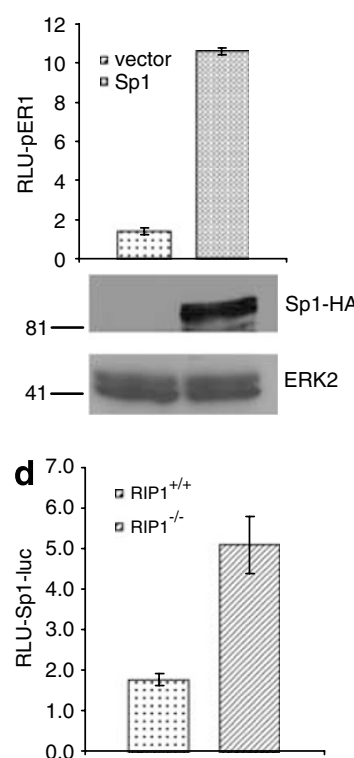

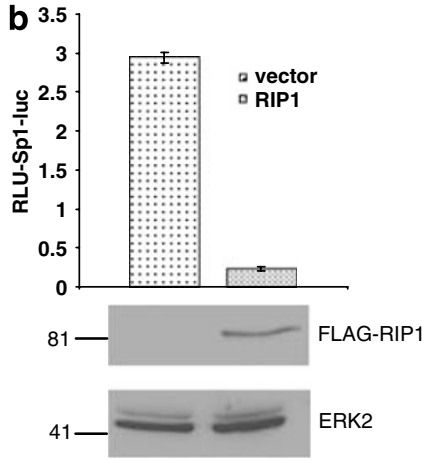

e

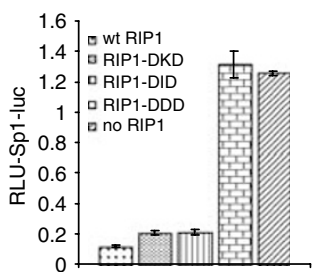

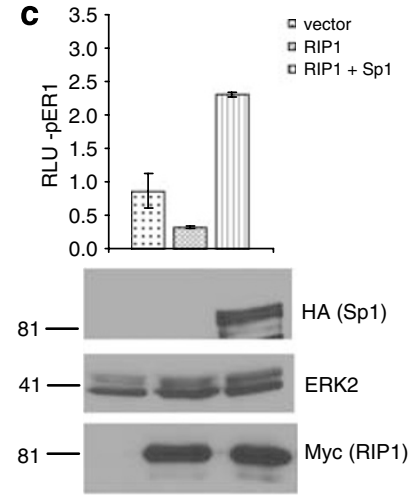

Figure 5 RIP1 regulates the EGFR promoter by inhibiting Sp1. (a) Expression of wild-type Sp1 increases the activity of the EGFR promoter (pER1-LUC). 293 Cells were transfected with the wild-type EGFR promoter linked to luciferase along with either empty vector or wild type HA-tagged Sp1 as described above. Sp1 expression was confirmed by Western blotting using HA antibodies. (b) 293 cells were transfected with a Sp1-LUC construct along with either empty vector or wild type RIP1-FLAG. Expression of RIP1 strongly inhibits the activity of Sp1. Expression of RIP1 was detected using FLAG antibodies. (c) Shows that overexpression of Sp1 overcomes the RIP1mediated suppression of the EGFR promoter (pER1-LUC). 293 cells were transfected with pER1-LUC and empty vector or Myc-tagged RIP1 along with either empty vector or wild type HA-tagged Sp1. Expression of Sp1 overcomes the RIP1-mediated suppression of the EGFR promoter. The lower panel shows expression of Sp1 and RIP1. (d) shows that Sp1 activity is increased in RIP1 ${ }^{-/-}$MEFs compared to RIP1 ${ }^{+/+}$MEFs. Sp1-LUC was transfected into MEFs followed by luciferase assays. (e) The effect of RIP1 mutation on Sp1-LUC in 293 cells. Expression of the RIP1 DDD (deletion of the death domain) fails to inhibit Sp1-LUC while other mutants are not impaired. At least three independent experiments were done in triplicate. Cotransfection of renilla luciferase was used as an internal control in all experiments. Error bars represent S.E.M. of triplicate determinations

Direct binding of recombinant RIP1 to Sp1 in vitro. To determine whether RIP1 and Sp1 interacted directly, we generated recombinant His-tagged RIP1. RIP1 was cloned into pBADMycHis vector, followed by transformation and induction. A lysate was prepared and induction of RIP1 was confirmed using Western blotting (data not shown). The HisRIP1 containing lysate was incubated with GST-Sp1 or GST alone bound to GST beads. The beads were subsequently washed and Western blotting was performed with RIP1 antibodies. The Western blot in Figure $8 d$ shows that recombinant RIP1 binds directly to GST-Sp1 but not to GST. Expression of GST-Sp1 was confirmed by Western blotting (Figure 8e).

\section{Discussion}

In this study, we describe a new link between the inflammatory/immune signaling pathways and growth factor signaling. We provide genetic evidence that RIP1, a key component of inflammatory and immune signaling pathways, regulates expression of the EGFR. Previous studies have reported a number of factors that influence activity of the EGFR promoter and expression of the receptor. ${ }^{22}$ Among the proteins known to activate the promoter are p53, IRF1, and Egr-1. ${ }^{23-26}$ Proteins known to repress the EGFR promoter include the Wilms' tumor suppressor (WT1), E1A, PML, and p63. ${ }^{20,22,27,28}$ However, the role of these proteins in regulation of EGFR expression in vivo is unclear.
We show that the loss of RIP1 from cells results in a substantial increase in EGFR levels and reconstitution of these cells results in a lowering of EGFR levels indicating a direct role for RIP1 in regulating EGFR levels. Northern blotting shows that RIP1 regulates EGFR at the MRNA level. RIP1 inhibits the activity of the EGFR promoter in reporter assays and loss of RIP1 results in increased EGFR promoter activity. Since RIP1 does not have a DNA binding domain, it is likely that RIP1 acts indirectly via a transcriptional regulator. Our data suggest that RIP1 interferes with the function of Sp1, an essential transcriptional activator of the EGFR promoter. RIP1 inhibits the activity of Sp1 in reporter assays and overexpression of $\mathrm{Sp} 1$ rescues the RIP1-mediated inhibition of the EGFR promoter. Loss of RIP1 results in an increase in Sp1 activity. Furthermore, RIP1 inhibits Sp1 binding to DNA. A previous study has reported the physical interaction of $\mathrm{Sp} 1$ with an unidentified protein with a molecular mass of $74 \mathrm{kDa}^{29}$ which inhibited the activity of Sp1. RIP1 has a molecular mass of $74 \mathrm{kDa}$ and appears to match the profile of this previously described inhibitor of Sp1. Importantly, inhibition of Sp1 function seems to be the major mechanism used to negatively regulate EGFR expression by a number of factors including PML, p63, and WT1 ${ }^{20,22,27}$ The exact mechanism of inhibition of Sp1 is unknown for any of these proteins, but like RIP1 they appear to inhibit Sp1 activity by forming a physical complex with it.

RIP1 is composed of a kinase, intermediate, and death domain. Death domains, in general, mediate interactions with 


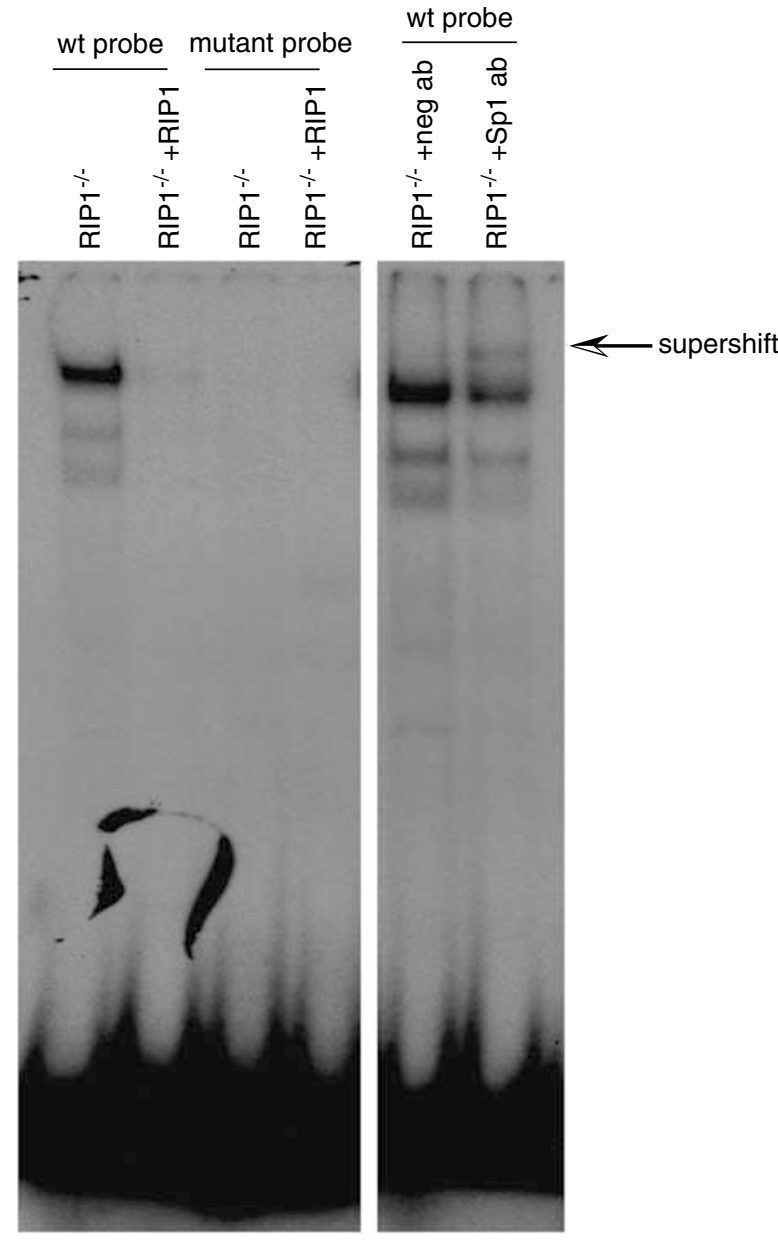

Figure 6 RIP1 expression impairs Sp1 DNA binding. Electrophoretic mobility shift assays of RIP1 $1^{-1-}$ or RIP1 ${ }^{-1-}$ cells expressing RIP1 (infection with RIP1 adenovirus for $24 \mathrm{~h}$ ) with the labeled Sp1 consensus oligonucleotide. No binding is detected with a mutant $\mathrm{Sp} 1$ oligonucleotide. For the supershift assay anti-Sp1 or control IgG was added to the binding reaction. The supershift shown in the right panel was done in a different part of the same gel shown in the left panel

other death domain-containing proteins. For example, the death domain of RIP1 mediates its interaction with death domains of FADD and TRADD. ${ }^{5}$ Our data suggest that the death domain of RIP1 plays a key role in regulation of EGFR expression.

RIP1, although mostly cytoplasmic, is also present in the nucleus. A previous study has shown that signaling molecules of the NF- $\kappa$ B signaling pathway such as TRAF2 and NIK shuttle constitutively between the cytoplasm and nucleus. ${ }^{30}$ Also, two other adapter proteins, TRADD and FADD, which (like RIP1) form complexes with the TNFR at the cell membrane, have also been localized to the nucleus. ${ }^{31-33}$ Thus, a nuclear localization of RIP1 is not surprising and would allow it to physically interact with nuclear Sp1.

Does NF- $\kappa$ B activation play a role in RIP1-mediated EGFR regulation? Previous studies have shown that NF- $\kappa$ B either has no effect or increases the activity of the EGFR promoter. ${ }^{34,35}$ Since RIP1 is a powerful activator of NF- $\kappa \mathrm{B}$, the repression of the EGFR promoter by RIP1 is unlikely to be mediated through activation of NF- $\kappa \mathrm{B}$. Furthermore, we find that a fragment of the EGFR promoter (NS, -153 to -19$)$ that lacks all five putative NF- $\kappa$ B binding sites spanning from -963 to $-231,{ }^{34}$ is fully repressed by RIP1 (Figure $3 \mathrm{~g}$ ).

Chronic inflammation promotes cancer, and it may seem paradoxical that RIP1, a key component of inflammatory signaling, should downregulate the EGFR. However, this finding is quite consistent with what is known about TNFR signaling which is remarkably context-dependent. TNF $\alpha$ has also been reported to either increase or decrease expression of the EGFR (depending on the cell type) but the mechanisms are poorly understood. ${ }^{36,37}$ The EGFR has multiple roles in development, normal physiology, as well as in diseases such as cancer, and RIP1 (which is widely expressed) could have a profound influence on any of these processes.

In a previous study, we showed that RIP1 forms a complex with the EGFR, and suggested that this may facilitate EGFRmediated NF- $\kappa$ B activation, suggesting a synergistic effect on signaling ${ }^{38}$ and promotion of cell survival. At this time, it is difficult to reconcile the previous data with the current study, which demonstrates that RIP1 negatively regulates EGFR expression. We would point out, however, that such complexity and seemingly contradictory responses are rather characteristic of both the TNFR and NF- $\kappa$ B-signaling pathways. ${ }^{13,39-41}$ This study provides a further insight into the interaction between the TNFR signaling pathway and the EGFR system by demonstrating that RIP1, a key component of TNFR signaling, is a powerful regulator of EGFR expression. Future studies will investigate whether RIP1 influences EGFR levels in development and/or cancer.

\section{Materials and Methods}

Plasmids and Cell lines. Wild type and deletion mutants of RIP1 domains named DKD (deletion of kinase domain), DID (deletion of intermediate domain), and DDD (deletion of death domain) were cloned into pcDNA3.1 with a C-terminal FLAG tag using standard molecular cloning techniques. An EGFR promoter luciferase construct was obtained from Dr. Alfred Johnson. ${ }^{22} \mathrm{~A}$ wild type EGFR promoter and mutants linked to luciferase was also obtained from Dr. Gordon Gill. ${ }^{19} \mathrm{HA}$-tagged Sp1 and Sp1-LUC and NEMO-Myc constructs were obtained from Dr. Pran Datta (Vanderbilt U) and Dr. Youn-Tae Kwok (UTSW). rip $1^{+/+} / \mathrm{tnfr}^{-{ }^{--}}$and rip $^{1-1}$ tnfr $1 /^{-1-}$ MEFs were generated as described previously. ${ }^{42}$ RIP1 knockout MEFs in a wild type TNFR background have been described previously. ${ }^{10}$ Immortalized wild type and rip $1^{-1-} 3 \mathrm{~T} 3$ cells lines were prepared according to established protocols. MDA-MB-468 and MCF7 cells were obtained from ATCC (Manassas, VA, USA). H157, HCC1569, HCC38, HCC3153, HCC2185, and HCC1937 cells were generously provided by Drs Cheryl Lewis and David Euhus (UTSW).

Antibodies, Western, and Northern blotting. EGFR (06-129) and Sp1 (07-645) antibodies were purchased from Upstate Biotechnology (Lake Placid, New York, USA) and used for Western blotting. RIP (610459) and PDGFR $\beta$ (554288) antibodies were purchased from BD Biosciences (San Jose, CA, USA). Cu/Zn superoxide dismutase antibodies (574597) were obtained from EMD Biosciences (San Diego, CA, USA). FLAG antibodies (M2) were purchased from Sigma (St. Louis, MO, USA) and from Cell Signaling Technology (\#2368, Danvers, MA, USA). FGFR3 (sc-123), Myc (9E10) and IGF-IR $\beta$ (sc-713) were purchased from Santa Cruz Biotechnology (Santa Cruz, CA, USA). Immunoprecipitation was performed on whole cell extracts. Immunoprecipitation and Western blotting were performed according to standard protocols and as we have described previously. ${ }^{38}$ Other antibodies used in this study include HA (12 CA5) obtained from Roche Applied Science (Indianapolis, IN, USA), His antibodies R930-25 from Invitrogen (Carlsbad, CA, USA), and phospho-ERK antibodies (\#9101) and Myc (\#2278) from Cell Signaling Technology. Northern blotting was conducted according to standard protocols. The probe was generated by using the EGFR gene as a template and 
a

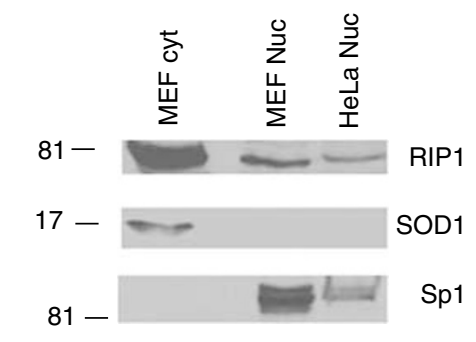

b
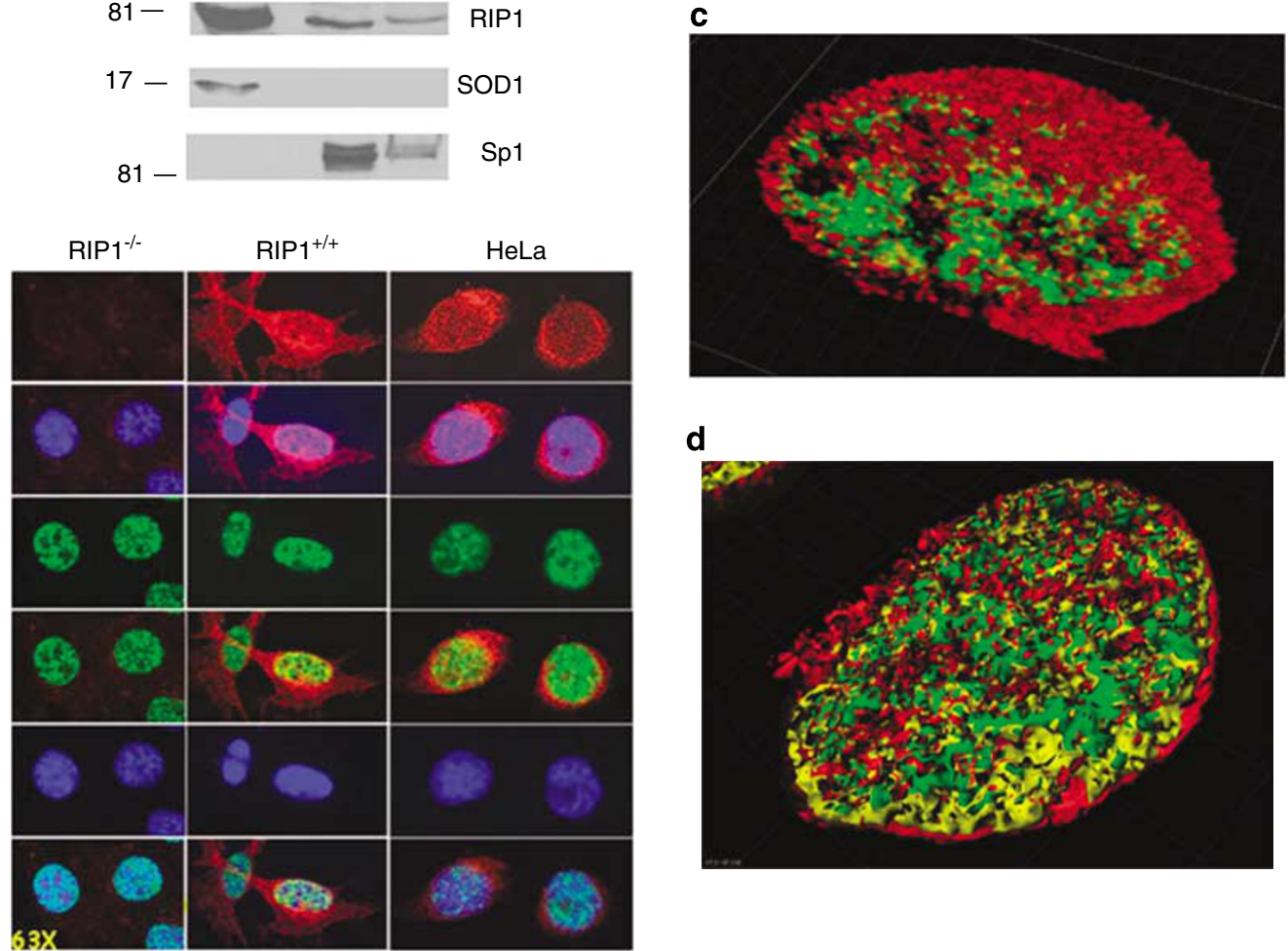

d

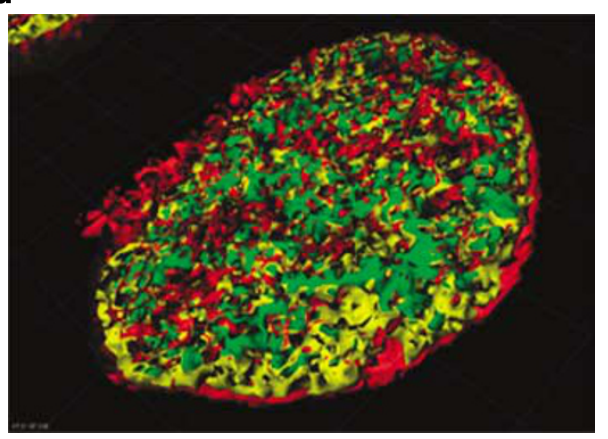

Figure 7 Presence of endogenous RIP1 in the nucleus. (a) Nuclear lysates from RIP1 ${ }^{+/+}$MEFs and from HeLa cells were prepared and subjected to Western blotting with RIP1 antibodies. As can be seen in the upper panel, RIP1 can be detected in the nucleus. To exclude contamination of nuclear extracts with cytoplasmic RIP1 we also probed with SOD (Cu/Zn superoxide dismutase) antibodies in the lower panel. While SOD can be detected in the cytoplasmic fraction (cyt), no SOD can be detected in the nuclear extracts (Nuc). Sp1 can be detected only in the nuclear extracts. (b) Immunofluorescent staining: Fluorescence microscopy of RIP1 $1^{-1-}, \mathrm{RIP} 1^{+1+}$ and HeLa cells to show cellular distribution of endogenous RIP1. As can be seen in the top panel RIP1 localizes to both the cytoplasm and the nucleus in MEFs and HeLa cells. No RIP1 can be detected in the RIP1 ${ }^{-l-}$ cells confirming the specificity of the RIP1 antibody. Sp1 is predominantly nuclear in both cell types. (c and d) Show the confocal snapshots in the volume and as a model respectively. The pictures show the distribution of RIP1 (red) and Sp1 (green) in the nucleus of RIP1 ${ }^{+l+}$ cells. The yellow spots show the overlap of the two proteins

probes were labeled with $\alpha^{32} \mathrm{P}$-dCTP using random priming. Equal loading of RNA was confirmed by stripping and reprobing the membrane for GAPDH.

Oligonucleotides. Oligonucleotides containing a potential Sp1-binding site were synthesized as follows: 0-Sp1, 5'-ATTCGATCGGGGCGGGGCGAGC-3' (sense) and 5'-GCTCGCCCCGCCCCGATCGAAT-3' (antisense); mutant 0-Sp1mut, $5^{\prime}$-ATTCGATCGtatttaGGCGAGC-3' (sense) and $5^{\prime}$-GCTCGCCtaaataCGA TCGAAT- $3^{\prime}$ (antisense); The complementary oligonucleotides were annealed and purified following the manufacturer's protocol. [ $\gamma^{-32}$ P]ATP was purchased from ICN Biochemicals (Irvine, CA, USA).

Transfection. 293 Cells were transfected using the calcium phosphate technique using standard protocols and expression of transfected genes was confirmed by Western Blotting. For transient transfection experiments, cells were harvested 24-48 h after transfection. MEFs were transfected using Lipofectamine reagent from Invitrogen (Carlsbad, CA, USA) according to the manufacturer's protocol.

Luciferase assays. 293 cells $\left(1.25 \times 10^{5}\right)$ or MEFs $\left(4 \times 10^{4}\right)$ were plated in 24-well dishes followed by transfection with either pER1-LUC or Sp1-LUC along with RIP1 plasmids or empty vector, using calcium phosphate or Lipofectamine respectively. A dual-luciferase reporter assay system was used according to the instructions of the manufacturer (Promega, Madison WI, USA). Firefly luciferase activity was measured in a luminometer and normalized on the basis of cotransfected Renilla luciferase activity.
Electrophoretic mobility shift assays. Nuclear extracts were prepared as described below. Probes (double-stranded oligonucleotides) containing the Sp1binding site were radiolabeled using T4 polynucleotide kinase and $\left[\gamma^{32}\right.$ P]ATP according to standard protocols. A typical binding reaction involved a $15 \mathrm{~min}$ preincubation with $5 \mu \mathrm{g}$ nuclear extract, $1.5 \mu \mathrm{g}$ poly dA-dT, $200 \mathrm{ng}$ single-stranded oligonucleotide, $20 \mathrm{mmol} / \mathrm{HEPES}-\mathrm{NaOH}$ (pH 7.6), $100 \mathrm{mmol} / \mathrm{I} \mathrm{NaCl}, 1 \mathrm{mmol} / \mathrm{IDTT}$, and $2 \%$ glycerol followed by a $20 \mathrm{~min}$ incubation with $20 \mathrm{fmol}$ radiolabeled probe. To assess the specificity of DNA protein-binding, radiolabeled mutant oligonucleotide was added instead of wild-type radiolabeled oligonucleotide to the binding reaction mixture. In the supershift analysis, Sp1-specific antibody $(1 \mu \mathrm{g})$ was added to the mixture and incubated for an additional $10 \mathrm{~min}$ at room temperature before electrophoresis. Normal rabbit IgG was used as control. Complexes were then resolved by electrophoresis for $90 \mathrm{~min}$ at $200 \mathrm{~V}$ on a $6 \%$ native polyacrylamide gel, dried, and processed for autoradiography.

Production of adenovirus-expressing RIP1. An adenoviral vector (first generation with $\mathrm{E} 1$ and $\mathrm{E} 3$ deleted) was used and wild type or RIP1 mutants were cloned into the vector. This resulted in a Tet operon-minimal CMV promoter-driven cassette in place of the AdE1 region. Ad-tTA (tetracycline controlled transactivator) was also prepared. An MOI of 50 was used in the experiments.

Expression of recombinant proteins and in vitro binding. pGEX1GST-Sp1 was transformed in BL21-competent cells and a culture was induced with IPTG. The induced culture was spun at 6000 r.p.m. for about $10 \mathrm{~min}$ and the pellet was dissolved in $4 \mathrm{ml}$ of ice cold PBS, sonicated in lysis buffer $(20 \mathrm{mM}$ Tris $\mathrm{HCl} \mathrm{pH}$ 


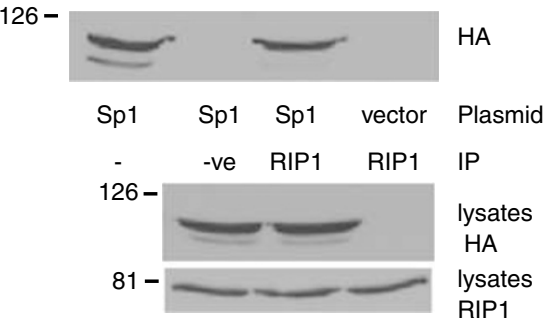

C $126-$

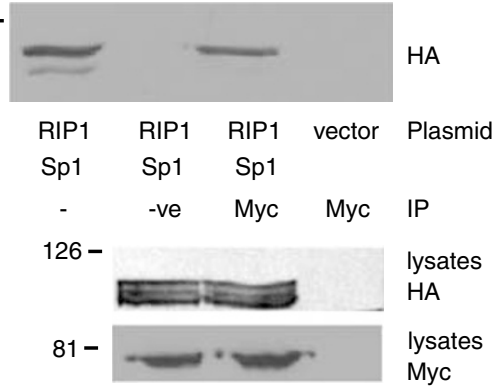

b

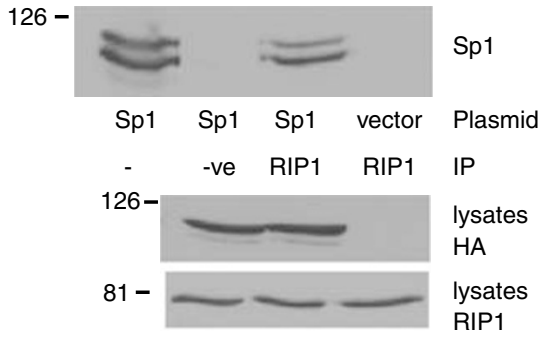

d GST GST-Sp1 Input 5\%

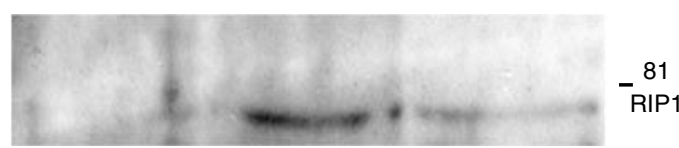

e

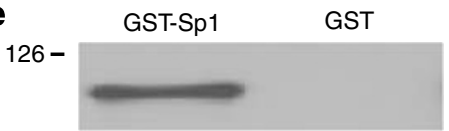

Sp1

Figure 8 RIP1 forms a physical complex with Sp1. (a) In this experiment, we transfected 293 cells with $10 \mu \mathrm{g}$ vector or Sp1-HA, followed by immunoprecipitation with a RIP1 antibody or with isotype matched negative control antibodies as shown. The Western blot was probed with HA antibodies. In the left lane 5\% of lysate from Sp1 overexpressing cells is loaded as a positive control. Prior to adding the immunoprecipitating antibody an aliquot was taken from each IP sample and equal amounts of the total lysate were probed for HA (middle panel) or RIP1 (lower panel) by Western blot. (b) Experimental conditions are similar to A except that Sp1 antibodies were used for immunoblotting in the upper panel. (c) 293 cells were transfected with either empty vector or RIP1-Myc plus Sp1-HA. Immunoprecipitation was performed using Myc antibodies or isotype matched control antibodies followed by immunoblotting with HA antibodies. In the left lane $5 \%$ of lysate from RIP1/Sp1 overexpressing cells is loaded as a positive control. Prior to adding the immunoprecipitating antibody an aliquot was taken from each IP sample and equal amounts of the total lysate was probed for HA (middle panel) or Myc (lower panel). (d) Shows a direct binding of His-tagged RIP1 to GST-Sp1 in an in vitro binding assay. RIP1 binds to GST-Sp1 but not to GST alone. (e) Shows expression of GST-Sp1

$7.5,500 \mathrm{mM} \mathrm{NaCl}, 0.1 \% \mathrm{NP} 40$ containing protease inhibitor) and centrifuged at 14000 r.p.m. for $30 \mathrm{~min}$ at $4^{\circ} \mathrm{C}$. The aliquots were stored at $-80^{\circ} \mathrm{C}$. For the binding of GST-Sp1 to the GST beads, $20 \mu$ l of GST beads was added to a $1.5 \mathrm{ml}$ tube and washed using TBS-Tween. For binding reactions, we added $300 \mu \mathrm{l}$ of the binding buffer (20 mM Tris HCl pH 7.5, $150 \mathrm{mM} \mathrm{NaCl}, 0.1 \% \mathrm{NP} 40,1 \% \mathrm{BSA}+$ complete protease inhibitor) followed by $500 \mu \mathrm{l}$ of the lysate supernatant. This was mixed gently on a nutator for $1 \mathrm{~h}$ at $4 \mathrm{C}$ followed by a wash in $1 \mathrm{ml}$ of washing buffer $(20 \mathrm{mM}$

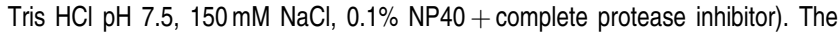
beads were washed three times. To confirm expression of GST-Sp1, proteins were eluted from beads followed by Western blotting with Sp1 antibodies.

RIP1 was cloned in $\mathrm{PBAD} / \mathrm{myc}$-His $\mathrm{C}$ vector (Invitrogen, Carlsbad, CA, USA) using standard molecular cloning techniques. Bacterial cultures were induced with $0.2 \%$ arabinose. An aliquot of the culture, before and after induction, was used to test the expression of RIP1 by Western blotting.

GST-Sp1 beads were incubated with His-RIP1 lysates for $1 \mathrm{~h}$ at $4^{\circ} \mathrm{C}$. This was followed by about $3-4$ washes using washing buffer. Following the washes, sample buffer was added to the beads and proteins were eluted and denatured followed by Western blotting.

Cell fractionation. Nuclear extracts were made from wild-type MEFs and HeLa cells using a nuclear extraction kit from Active Motif (Carlsbad, CA, USA) according to the manufacturer's instructions with one modification. To eliminate cytoplasmic contamination of nuclear lysates, the nuclear pellet was washed three times in $1 \times$ hypotonic buffer solution. To detect cytoplasmic contamination of nuclear extracts, we used antibodies to $\mathrm{Cu} / \mathrm{Zn}$ superoxide dismutase (SOD) in Western blots of nuclear extracts.

Fluorescent immunostaining and confocal microscopy. Wild-type RIP1 knockout fibroblasts and HeLa cells were grown on chamber slides to about $70 \%$ confluence. Cells were fixed in ice-cold methanol for $60 \mathrm{~min}$ at $-20{ }^{\circ} \mathrm{C}$ then permeabilized with Triton X-100 (0.5\% v/v in PBS) for 10 min on ice. Non-specific binding sites were blocked with $5 \%$ normal goat serum (in PBS) for $1 \mathrm{~h}$ at room temperature. Cells were exposed to anti-Sp1 rabbit polyclonal (Upstate; dilution $1: 75$ in PBS/5\% goat serum), RIP1 mouse monoclonal (dilution $1: 150$ in $\mathrm{PBS} / 5 \%$ goat serum) antibodies for $2 \mathrm{~h}$ at room temperature, washed thrice with wash buffer (1\% BSA/phosphate-buffered saline), and subsequently stained with Alexa Fluor 488-conjugated goat anti-rabbit and Rhodamine Red-conjugated goat anti mouse secondary antibodies (Molecular Probes, Eugene, OR, USA; dilution 1:1000 in $\mathrm{PBS} / 2.5 \%$ goat serum $/ 1 \% \mathrm{BSA}$ ) for $1 \mathrm{~h}$ at room temperature. Cells were then washed five times with wash buffer and mounted using Vectashield (mounting medium with 4,6 diamidino-2-phenylindole from Vector Laboratory, Burlingame, CA, USA). Images were acquired using an Apotome Zeiss microscope and Axiovision software. Apotome Zeiss system provides an optical slice view reconstructed from fluorescent samples, using a series of 'grid projection' acquisitions. To allow direct comparisons, all the cells were processed simultaneously, and all the images were obtained using the same parameters (brightness, contrast, etc.).

For confocal microscopy, z-sections were done with an optical section spacing of 0.38 microns with a Zeiss LSM 510 microscope. The optical section thickness was kept the same for both green and red. Each image in the stack was captured with an optimum slice thickness using a pinhole size of airy 1 for the higher wavelength (red). These were saved in imaris 5.5.2 as ims file. The background subtraction was done before looking for colocalization. The analysis for colocalization was done using Imaris 3D software.

Statistical analysis. To determine the statistical significance between different samples, we used student's $t$-test. A $P$-value of $<0.05$ was considered statistically significant.

Acknowledgements. This work was supported by NIH Grant CA 78741 to $\mathrm{AH}$ and by funding from the Annette Strauss Center in Neuro-oncology at UTSW, and from the Children's Brain Tumor Foundation of the Southwest. We thank Dr. Brian Seed for RIP1 constructs, Dr. Alfred Johnson and Dr. Gordon Gill for EGFR promoter constructs, Dr. Pran Datta for Sp1-LUC and wild type Sp1, Dr. Jane Azizkhan-Clifford and Dr. Jonathon Horowitz for GST-Sp1 constructs, Dr. Cheryl Lewis and Dr. David Euhus for breast cancer cell lines. We thank Abhijit Bugde, Dr. Kate Luby-Phelps and Dr. Krishna Puttaparthi for help with confocal microscopy and 
immunofluorescent staining. We thank Dr. Benjamin Neel for helpful discussions and advice. The authors declare no financial conflict of interest.

1. Balkwill F, Mantovani A. Inflammation and cancer: back to Virchow? Lancet 2001; 357 539-545.

2. Karin M, Greten FR. NF-kappaB: linking inflammation and immunity to cancer developmen and progression. Nat Rev Immunol 2005; 5: 749-759.

3. Blume-Jensen P, Hunter T. Oncogenic kinase signalling. Nature 2001; 411: 355-365.

4. Lollini PL, Cavallo F, Nanni P, Forni G. Vaccines for tumour prevention. Nat Rev Cancer 2006; 6: 204-216.

5. Meylan E, Tschopp J. The RIP kinases: crucial integrators of cellular stress. Trends Biochem Sci 2005; 30: 151-159.

6. Stanger BZ, Leder P, Lee TH, Kim E, Seed B. RIP: a novel protein containing a death domain that interacts with Fas/APO-1 (CD95) in yeast and causes cell death. Cell 1995; 81 513-523.

7. Festjens N, Vanden Berghe T, Cornelis S, Vandenabeele P. RIP1, a kinase on the crossroads of a cell's decision to live or die. Cell Death Differ 2007; 14: 400-410.

8. Hsu H, Huang J, Shu HB, Baichwal V, Goeddel DV. TNF-dependent recruitment of the protein kinase RIP to the TNF receptor-1 signaling complex. Immunity 1996; 4: 387-396.

9. Ting AT, Pimentel-Muinos FX, Seed B. RIP mediates tumor necrosis factor receptor 1 activation of NF-kappaB but not Fas/APO-1-initiated apoptosis. EMBO J 1996; 15 : 6189-6196

10. Kelliher MA, Grimm S, Ishida Y, Kuo F, Stanger BZ, Leder $P$. The death domain kinase RIP mediates the TNF-induced NF-kappaB signal. Immunity 1998; 8: 297-303.

11. Meylan E, Burns K, Hofmann K, Blancheteau V, Martinon F, Kelliher M et al. RIP1 is an essential mediator of Toll-like receptor 3-induced NF-kappa B activation. Nat Immunol 2004; 5: 503-507

12. Hur GM, Lewis J, Yang Q, Lin Y, Nakano H, Nedospasov S et al. The death domain kinase RIP has an essential role in DNA damage-induced NF-kappa B activation. Genes Dev 2003; 17: 873-882.

13. Pimentel-Muinos FX, Seed B. Regulated commitment of TNF receptor signaling: molecular switch for death or activation. Immunity 1999; 11: 783-793.

14. Johnson AC, Ishii S, Jinno Y, Pastan I, Merlino GT. Epidermal growth factor receptor gene promoter. Deletion analysis and identification of nuclear protein binding sites. J Biol Chem 1988; 263: 5693-5699.

15. Hudson LG, Santon JB, Gill GN. Regulation of epidermal growth factor receptor gene expression. Mol Endocrinol 1989; 3: 400-408.

16. Kageyama R, Merlino GT, Pastan I. Epidermal growth factor (EGF) receptor gene transcription. Requirement for Sp1 and an EGF receptor-specific factor. J Biol Chem 1988; 263: 6329-6336.

17. DiMario JX. Activation and repression of growth factor receptor gene transcription (Review). Int J Mol Med 2002; 10: 65-71.

18. Krappmann D, Hatada EN, Tegethoff S, Li J, Klippel A, Giese K et al. The I kappa B kinase (IKK) complex is tripartite and contains IKK gamma but not IKAP as a regular component. J Biol Chem 2000; 275: 29779-29787.

19. Hudson LG, Thompson KL, Xu J, Gill GN. Identification and characterization of a regulated promoter element in the epidermal growth factor receptor gene. Proc Natl Acad Sci USA 1990; 87: 7536-7540.

20. Vallian S, Chin KV, Chang KS. The promyelocytic leukemia protein interacts with Sp1 and inhibits its transactivation of the epidermal growth factor receptor promoter. $\mathrm{Mol}$ Cell Biol 1998; 18: 7147-7156.

21. Micheau O, Tschopp J. Induction of TNF receptor I-mediated apoptosis via two sequentia signaling complexes. Cell 2003; 114: 181-190.

22. Nishi H, Senoo M, Nishi KH, Murphy B, Rikiyama T, Matsumura $Y$ et al. p53 Homologue p63 represses epidermal growth factor receptor expression. J Biol Chem 2001; 276: $41717-41724$.
23. Ludes-Meyers JH, Subler MA, Shivakumar CV, Munoz RM, Jiang P, Bigger JE et al. Transcriptional activation of the human epidermal growth factor receptor promoter by human p53. Mol Cell Biol 1996; 16: 6009-6019.

24. Sheikh MS, Carrier F, Johnson AC, Ogdon SE, Fornace Jr AJ. Identification of an additional p53-responsive site in the human epidermal growth factor receptor gene promotor. Oncogene 1997; 15: 1095-1101.

25. Rubinstein YR, Proctor KN, Bergel M, Murphy B, Johnson AC. Interferon regulatory factor1 is a major regulator of epidermal growth factor receptor gene expression. FEBS Lett 1998; 431: 268-272.

26. Nishi $\mathrm{H}$, Nishi $\mathrm{KH}$, Johnson AC. Early growth response-1 gene mediates up-regulation of epidermal growth factor receptor expression during hypoxia. Cancer Res 2002; 62 . 827-834.

27. Englert $C$, Hou X, Maheswaran S, Bennett P, Ngwu C, Re GG et al. WT1 suppresses synthesis of the epidermal growth factor receptor and induces apoptosis. EMBO J 1995; 14: $4662-4675$.

28. Prudenziati $M$, Sirito $M$, van Dam $H$, Ravazzolo R. Adenovirus E1A down-regulates the EGF receptor via repression of its promoter. Int J Cancer 2000; 88: 943-948.

29. Murata Y, Kim HG, Rogers KT, Udvadia AJ, Horowitz JM. Negative regulation of Sp1 transactivation is correlated with the binding of cellular proteins to the amino terminus of the Sp1 trans-activation domain. J Biol Chem 1994; 269: 20674-20681.

30. Birbach A, Gold P, Binder BR, Hofer E, de Martin R, Schmid JA. Signaling molecules of the NF-kappa B pathway shuttle constitutively between cytoplasm and nucleus. J Biol Chem 2002; 277: 10842-10851.

31. Gomez-Angelats M, Cidlowski JA. Molecular evidence for the nuclear localization of FADD. Cell Death Differ 2003; 10: 791-797.

32. Screaton RA, Kiessling S, Sansom OJ, Millar CB, Maddison K, Bird A et al. Fas-associated death domain protein interacts with methyl-CpG binding domain protein 4: a potential link between genome surveillance and apoptosis. Proc Natl Acad Sci USA 2003; 100: 5211-5216.

33. Morgan M, Thorburn J, Pandolfi PP, Thorburn A. Nuclear and cytoplasmic shuttling of TRADD induces apoptosis via different mechanisms. J Cell Biol 2002; 157: 975-984.

34. Nishi H, Neta G, Nishi KH, Akers LM, Rikiyama T, Proctor KN et al. Analysis of the epidermal growth factor receptor promoter: the effect of nuclear factor-kappaB. Int J Mol Med 2003; 11: 49-55.

35. Tao YG, Tan YN, Liu YP, Song X, Zeng L, Gu HH et al. Epstein-Barr virus latent membrane protein 1 modulates epidermal growth factor receptor promoter activity in a nuclear factor kappa B-dependent manner. Cell Signal 2004; 16: 781-790.

36. Novotny-Smith CL, Zorbas MA, Mclsaac AM, Irimura T, Boman BM, Yeoman LC et al. Down-modulation of epidermal growth factor receptor accompanies TNF-induced differentiation of the DiFi human adenocarcinoma cell line toward a goblet-like phenotype. J Cell Physiol 1993; 157: 253-262.

37. Kalthoff H, Roeder C, Brockhaus M, Thiele HG, Schmiegel W. Tumor necrosis factor (TNF) up-regulates the expression of $\mathrm{p} 75$ but not p55 TNF receptors, and both receptors mediate, independently of each other, up-regulation of transforming growth factor alpha and epidermal growth factor receptor mRNA. J Biol Chem 1993; 268: 2762-2766.

38. Habib AA, Chatterjee S, Park SK, Ratan RR, Lefebvre S, Vartanian T. The epidermal growth factor receptor engages receptor interacting protein and nuclear factor-kappa $B$ (NF-kappa B)-inducing kinase to activate NF-kappa B. Identification of a novel receptortyrosine kinase signalosome. J Biol Chem 2001; 276: 8865-8874.

39. Wajant H, Pfizenmaier K, Scheurich P. Tumor necrosis factor signaling. Cell Death Differ 2003; 10: 45-65.

40. Perkins ND, Gilmore TD. Good cop, bad cop: the different faces of NF-kappaB. Cell Death Differ 2006; 13: 759-772.

41. Janes KA, Gaudet S, Albeck JG, Nielsen UB, Lauffenburger DA, Sorger PK. The response of human epithelial cells to TNF involves an inducible autocrine cascade. Cell 2006; 124: 1225-1239.

42. Cusson N, Oikemus S, Kilpatrick ED, Cunningham L, Kelliher M. The death domain kinase RIP protects thymocytes from tumor necrosis factor receptor type 2-induced cell death. J Exp Med 2002; 196: 15-26.

\section{Supplementary Information accompanies the paper on Cell Death and Differentiation website (http://www.nature.com/cdd)}

